\title{
Micro-usinage par laser à excimères
}

D. Damiani

Industrial Laser Partner, BP. 7, 87310 Saint Laurent-sur-Gorre, France

\begin{abstract}
Excimer laser boots the performances for material processing and micromachining applications. Because of short emission wavelength (in the UV range), short pulse duration (15ns) and high peak power $\left(>100 \mathrm{MW} / \mathrm{cm}^{2}\right)$ during laser emission; thermals effects during laser-material interaction are negligible. With appropriate beam delivery systems we perfome the micromachining of any kind of material (polymers, glasses, metals, ceramics...).

We show examples of excimer laser micromachining or engraving and especially micromachining of "hard" and "ultra-hard" materials with aspect ratio as high as 60 and hole diameter as small as $3 \mu \mathrm{m}$.
\end{abstract}

\section{INTRODUCTION}

L'usinage par laser à excimères est un nouveau procédé en plein développement.

Le laser Ultra Violet à Excimères permet de réaliser des usinages à l'échelle du micron, repoussant ainsi les limites dimensionnelles des procédés d'usinage traditionnels.

Le traitement par laser Ultra Violet s'applique à tous les matériaux (polymères, métaux, verres, céramiques, alliages, composites...).

Compte tenu des caractéristiques des lasers à Excimères (émission dans VUltra Violet, impulsion très brève et forte densité de puissance), les effets thermiques durant linteraction laser-matière sont négligeables.

La maîtrise de la mise en forme du faisceau laser permet le micro-usinage de tous les matériaux avec des vitesses élevées compatibles avec les demandes industrielles, tout en garantissant la précision et la reproductibilité de l'usinage ou de la gravure.

Ainsi, l'utilisation du laser Ultra Violet à Excimères n'est plus limitée seulement ả la gravure ou au perçage de polymère où le flux laser est inférieur au Joule/ $\mathrm{cm}^{2}$. 


\section{LE LASER A EXCIMERES}

\subsection{Caractéristiques.}

Le laser à excimères a été annoncé dès $1960 / 1 /$ et les premières applications datent de $1972 / 2 /$. Le mot "excimère" est la contraction des mots anglais "excited dimer" désignant ainsi la formation d'une molécule d'halogénure de gaz rare stable dans son état excitée (l'état fondamental est dissocié).

Suivant le mélange employé (Ar-F, $\mathrm{Kr}-\mathrm{F}, \mathrm{Xe}-\mathrm{Cl}$ ), la longueur d'onde d'émission est différente. Les longueurs d'onde d'émission se situent dans l'ultraviolet proche ou lointain (Cf. Table 2.1).

L'énergie disponible durant l'impulsion laser est très importante soit une puissance crête de plusieurs dizaines de $\mathrm{MW} / \mathrm{cm}^{2}$ pendant une impulsion courte (\#10-20ns). La puissance moyenne peut dépasser le kilowatt $/ 3 /$.

$\mathrm{La}$ fréquence de tir peut atteindre $1 \mathrm{kHz}$.

La dimension du faisceau est importante et généralement de forme rectangulaire $\left(10 \times 30 \mathrm{~mm}^{2}\right)$. La répartition en énergie est homogène dans tout le faisceau (créneau dans une direction et gaussienne dans l'autre)

La durée de vie du mélange gazeux est fortement liée à la nature et la qualité du mélange gazeux, aux conditions opératoires et aux matériaux qui constituent la cavité laser. Des progrès très récents $/ 4 /$ ont permis d'atteindre une durée de vie de $10^{9}$ tirs pour un mélange $\mathrm{KrF}$ soit un gain de plus de 10 .

Table 2.1 : Príncipales caractéristiques des lasers à excimères.

\begin{tabular}{|l|l|l|l|}
\hline $\begin{array}{l}\text { Halogénures de gaz } \\
\text { rares }\end{array}$ & $\begin{array}{l}\text { Longueur } \\
\text { d'onde (nm) }\end{array}$ & $\begin{array}{l}\text { Durée de l'impulsion } \\
\text { (ns) }\end{array}$ & $\begin{array}{l}\text { Energie par } \\
\text { impulsion (m) }\end{array}$ \\
\hline F2 & 157 & $6-18$ & $10-60$ \\
\hline $\mathrm{ArF}$ & 193 & $10-25$ & $10-650$ \\
\hline $\mathrm{KrF}$ & 248 & $12-60$ & $100-2000$ \\
\hline $\mathrm{XeCl}$ & 308 & $10-250$ & $100-15000$ \\
\hline $\mathrm{XeF}$ & 351 & $10-35$ & $10-650$ \\
\hline $\begin{array}{l}\text { Halogénures de gaz } \\
\text { rares }\end{array}$ & $\begin{array}{l}\text { Fréquence de tir } \\
\text { max (Hz) }\end{array}$ & $\begin{array}{l}\text { Puissance moyenne } \\
(\mathrm{W})\end{array}$ & $\begin{array}{l}\text { Durée de vie } \\
\text { (tirs) }\end{array}$ \\
\hline $\mathrm{F2}$ & 100 & $2-6$ & $4 \mathrm{E}+04$ \\
\hline ArF & 300 & $2-70$ & $20 \mathrm{E}+06$ \\
\hline $\mathrm{KrF}$ & 1000 & $3-200$ & $1 \mathrm{E}+09$ \\
\hline $\mathrm{XeCl}$ & 1000 & $3-1000$ & $2 \mathrm{E}+07$ \\
\hline $\mathrm{XeF}$ & 1000 & $2-20$ & $3 \mathrm{E}+06$ \\
\hline
\end{tabular}




\subsection{Interaction laser à excimères - matériau}

L'étude de l'interaction laser - matériau permet de distinguer de façon très schématique deux cas extrêmes d'interaction :

* interacticn photo-thermiqe

* interaction photochimique

\subsubsection{Interaction photo-thermique}

L'interaction de type thermique dont un exemple type concerne linteraction entre le rayonnement d'un laser $\mathrm{CO}_{2}$ et un métal se justifie par le fait que l'énergie du photon incident $\left(0.12 \mathrm{eV}\right.$ pour un laser $\mathrm{CO}_{2}$ à $\left.\lambda=10.6 \mu \mathrm{m}\right)$ est faible vis à vis de l'énergie de cohésion du matériau (quelques $\mathrm{eV}$ ). Ce dernier est chauffé très rapidement et si la densité de puissance est suffisante, on atteint la vaporisation. Le laser est plutôt une source de chaleur. Macroscopiquement, ceci se traduit par :

* absorption du faisceau et chauffage par conduction thermique de la cible.

* fusion de la cible.

* vaporisation de la matière avec éventuellement apparition d'un plasma.

\subsubsection{Interaction photochimique}

Un exemple de ce type d'interaction concerne linteraction entre le rayonnement d'un laser à excimères et un polymère. Dans ce cas l'énergie du photon ultraviolet est comparable à l'énergie de cohésion du matériau (un laser KrF émettant à $\lambda=248 \mathrm{~nm}$ correspond à un photon ayant une énergie de $5 \mathrm{eV}$ ). L'énergie du photon est suffisante pour rompre les liaisons inter atomiques du polymère. Si la densité de puissance appliquée au matériau atteint une valeur seuil pour briser un nombre suffisant de liaisons le matériau passe directement de la phase solide à la phase gazeuse. La matière est ejectée perpendiculairement à la surface.

L'interaction photochimique se résume en trois étapes :

* absorption du rayonnement laser

* ruptures des liaisons de valence

* ejection de matière.

L'énorme intérêt de cette interaction est qu'elle se fait sans élévation notable de la température de la cible car la profondeur de pénétration du rayonnement U.V. n'est que de quelques dizaines de nanomètres (pour un rayonnement infrarouge la profondeur est de l'ordre du micron). La faible durée de l'impulsion d'un laser à excimères minimise également la zone affectée thermiquement.

De plus, la plupart des matériaux absorbent d'avantage dans l'ultraviolet que dans linfrarouge.

\section{APPLICATIONS}

Les caractéristiques des lasers à excimères (émission dans l'ultraviolet, impulsion brève et forte densité de puissance) permettent de travailler tous les matériaux et plus particulièrement les polymères et les matériaux "fragiles" (verres, silicium monocristallin etc.).

La résolution ultime des usinages est de l'ordre de la longueur d'onde d'émission soit $0.25 \mu \mathrm{m}$ pour un laser $\mathrm{KrF}$ par exemple. 
Les principales applications industrielles concernent pour le moment linsolation de résine photosensible type PMMA utilisée en micro électronique (ou la gravure directe dans certains cas). Pour ce type d'application le laser à excimères est particulièrement bien adapté dans la mesure où la grande taille du faisceau permet de traiter des surfaces importantes (plusieurs $\mathrm{mm}^{2}$ à plusieurs $\mathrm{cm}^{2}$ ). Cependant les énergies misent en jeu sont faibles $\left(<1 \mathrm{~J} / \mathrm{cm}^{2}\right.$ au niveau du polymère).

Dans ce qui suit nous allons présenter un certain nombre d'applications :

* des applications "basse fluence" : marquage et gravure de matériaux tendres (polymères par exemple).

* des applications "haute fluence" pour l'usinage de matériau "durs et "ultradurs" (métaux, verres, céramiques, composites etc.).

\subsection{Mise en forme du faisceau laser}

Suivant le type d'application la mise en forme du faisceau laser est différente. Trois méthodes existent :

* masque de contact : un masque représentant un motif quelconque (traits, ouvertures de toutes formes) est plaqué ou situé à faible distance du matériau à traiter. Le faisceau laser irradie l'ensemble masque + matériau. Le déplacement du faisceau laser ou de l'ensemble masque + matériau permet de traiter des grandes surfaces. pièce à traiter.

* projection de masque : un système de lentilles fait limage d'un masque sur la

* faisceau focalisé par une lentille comme pour l'usinage par laser YAG ou $\mathrm{CO}_{2}$.

\subsection{Marquage}

Les lasers à excimères permettent de réaliser des marquages sur une multitude de matériaux avec une qualité bien supérieure aux autres techniques. Par exemple, il est possible de marquer un polymère par modification de sa couleur sans graver le matériau. Le changement de couleur est dû à un changement des liaisons inter-atomiques d'un pigment photosensible au rayonnement ultra violet.

La plupart du temps les énergies à mettre en oeuvre sont faibles $\left(\# 50 \mathrm{~mJ} / \mathrm{cm}^{2}\right) \mathrm{ce}$ qui permet de marquer des grandes surfaces (plusieurs dizaines de $\mathrm{mm}^{2}$ ). De plus, dans certains cas une impulsion laser suffit pour provoquer le changement de couleur. Le marquage à la volée est donc possible.

Ce type de marquage présente l'énorme avantage d'être inviolable du fait de sa qualité (la définition des marquages est de l'ordre du micron) et surtout parce que la suppression du marquage implique la destruction du support (le marquage est dans la masse du matériau).

Cette technique est utilisée notamment pour le marquage de câbles pour l'aéronautique, sur des prothèses médicales.

\subsection{Gravure}

\subsubsection{Grovure sélective}

La maîtrise de l'énergie du faisceau laser et du nombre d'impulsions permet de réaliser des gravures sélectives de matériaux multicouches.

La figure 3.1 montre un microcircuit protégé par une couche de Kapton de $15 \mu \mathrm{m}$ d'épaisseur dans laquelle a été pratiqué une ouverture carrée de $100 \mu \mathrm{m}^{2}$ sans altérer le conducteur en aluminium de $8000 \AA$ d'épaisseur. Les ouvertures (ou "pads") ainsi réalisées permettent la connexion du microcircuit aux fils d'alimentation ou de données. 
C'ette méthode de gravure directe permet d'éliminer les étapes d'insolation de ressine photosensible et de développement par voie chimique utilisées en microelectronique.

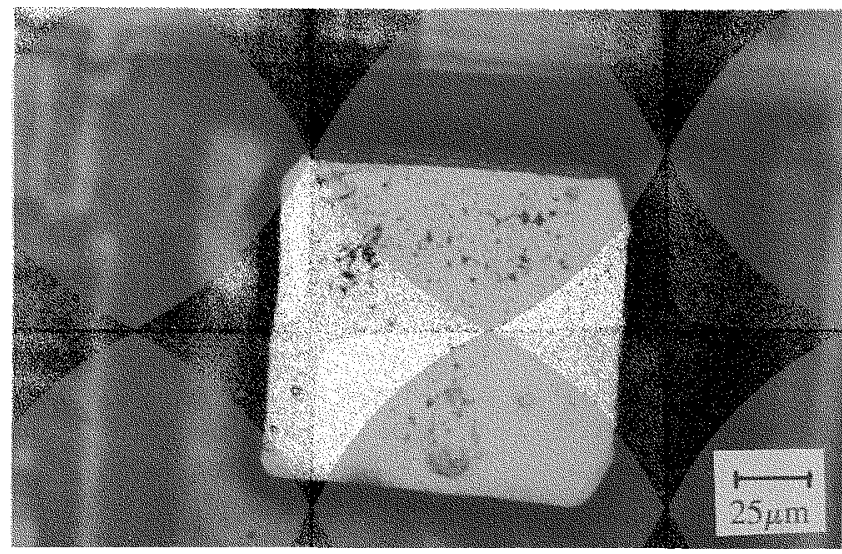

Figure 3.1 : Ouverture de $100 \times 100 \mu \mathrm{m}^{2}$ sur microcircuit.

\subsubsection{Gravure par Imagerie}

Par projection de masque il est possible de graver directement un motif complexe (Cf. Figure 3.2). La profondeur gravee est fonction du type de matériau, de la fluence laser et du nombre de tirs. Suivant les conditions expérimentales il est possible de contrôler l'état de surface de la gravure. Ainsi sur du verre ou du $\mathrm{ZnS}$ ou $\mathrm{ZnSe}$ (matériaux employés pour les optiques infrarouges) il est possible d'obtenir des gravures avec des rugosités d'environ $100 \AA$. Inversement, la rugosité peut être multipliée par10 sur des polymères par exemple.

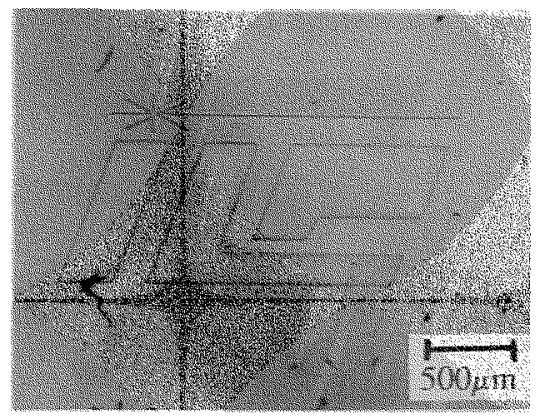

Figure 3.2 : Gravure sur verre. Dimension du motif : $2 \times 1 \mathrm{~mm}^{2}$.

\subsubsection{Gravure de réseanx}

La gravure de réseaux à pas fins (pas inférieur à $2 \mu \mathrm{m}$ ) est réalisée par interférences entre 2 faisceaux issus du même laser (Cf. figure 3.3). Cette méthode est 
particulièrement intéressante pour la réalisation de réseaux de Bragg sur fibre optique pour la fabrication de capteurs ou de coupleurs à fibre optique. Une impulsion laser suffit pour réaliser le réseau et permet un marquage à la volée d'une multitude de réseaux sur une même fibre. Des rẻseaux peuvent également être gravés sur des polymères ou du silicium.

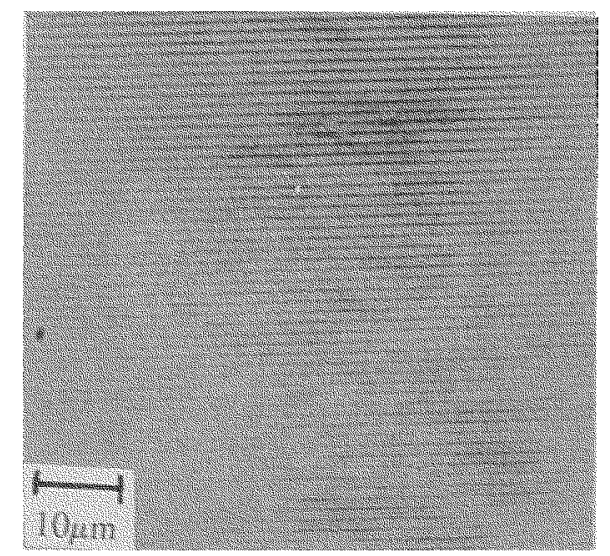

Figure 3.3 : Réseau sur verre. Pas du réseau : $1,1 \mu \mathrm{m}$

\subsection{Perçage, Découpe}

Dans ce qui suit nous présentons des exemples d'usinage de matériaux "durs" et "ultra-durs" avec des rapports épaisseurs sur diamètres supẻrieurs à 10.

La figure 3.4 montre l'absence d'effets thermiques l'ors de l'usinage d'alumine par exemple. Les trous réalisés par laser YAG ì $1.06 \mu$ m montrent la présence de matériau fondu autour du trou ( $\mathrm{Cf}$. figure 3.4.6). Par laser à excimères labsence dieffets thermiques ellinuine également les contraintes mécaniques et autorise lusingge sur le bord sans gểnérer de micro-fissuration du matérian (Cf. figure 3,4.a).
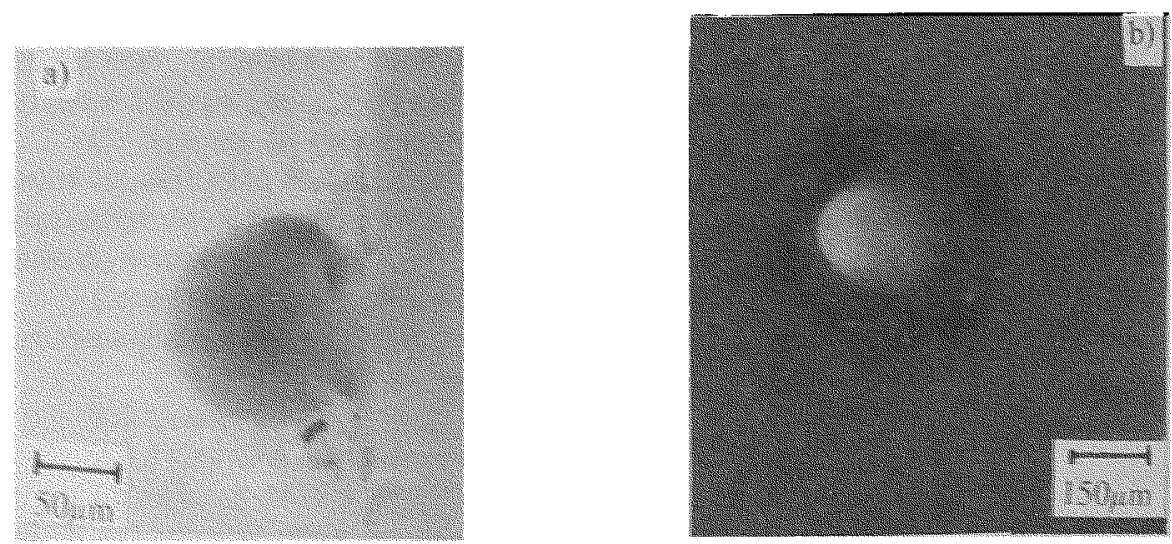

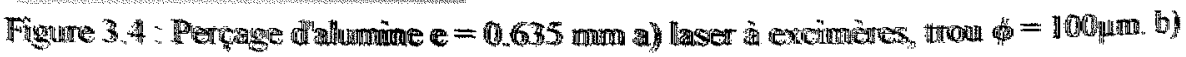
baser YAG $\lambda=1.06$ tum, trou $\phi=200$ num.

Les dimensions caracteristique des ussimages sont: 
- Trous circulaires ou non, débouchants ou non. Les diamètres de perçage sont compris entre $3 \mu \mathrm{m}$ et $500 \mu \mathrm{m}$.

- Epaisseur maximale de perçage jusqu'à $6 \mathrm{~mm}$

- Rapport épaisseur sur diamètre pouvant atteindre 60.

\subsubsection{Diaphragmes, fentes calibrées}

La figure 3.5 montre la réalisation d'une grille de trous calibrés de $7 \mu \mathrm{m}$ de diamètre au pas de $40 \mu \mathrm{m}$ sur du tungstène de $10 \mu \mathrm{m}$ d'épaisseur. L'ensemble des trous est réalisé en même temps. Par cette méthode il est possible de réaliser rapidement une grille de micro-trous pour la filtration. De même, le laser à excimères permet de réaliser des fentes pour la spectroscopie optique ou $X$.

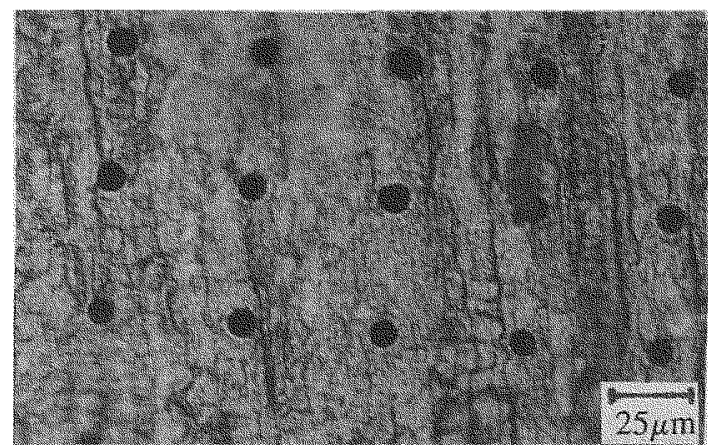

Figure 3.5: Tungstène $\mathrm{e}=10 \mu \mathrm{m}$. Trous de $7 \mu \mathrm{m}$ au pas de $40 \mu \mathrm{m}$

\subsection{Perçage "profond"}

Lorsque la fluence laser est très importante il est possible de percer tous les matériaux sur des épaisseurs importantes. Le perçage peut être non débouchant. Dans ces conditions les vitesses de perçages varient du micron à plusieurs microns par tir laser. Sur la face d'entrée du perçage le plasma, bien que très intense, ne fait pas écran au rayonnement laser (comme c'est le cas lors de l'interaction laser $\mathrm{CO}_{2}$-métal à haut flux laser $15 /$ ). La présence du plasma se traduit essentiellement par une augmentation du diamètre d'entrée ( $\mathrm{Cf}$. figure 3.7). Cependant le perçage cylindrique sur de très grande épaisseur est possible comme le montre la figure 3.8. Le rapport épaisseur sur diamètre est de 60 .

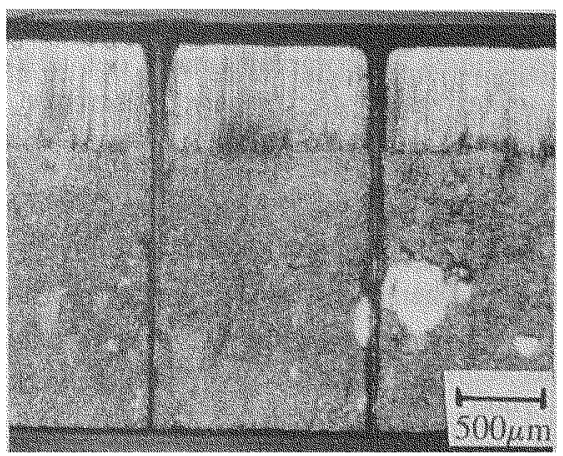

Figure 3.7 : Perçage d'acier inoxydable de $2 \mathrm{~mm}$ d'épaisseur. 


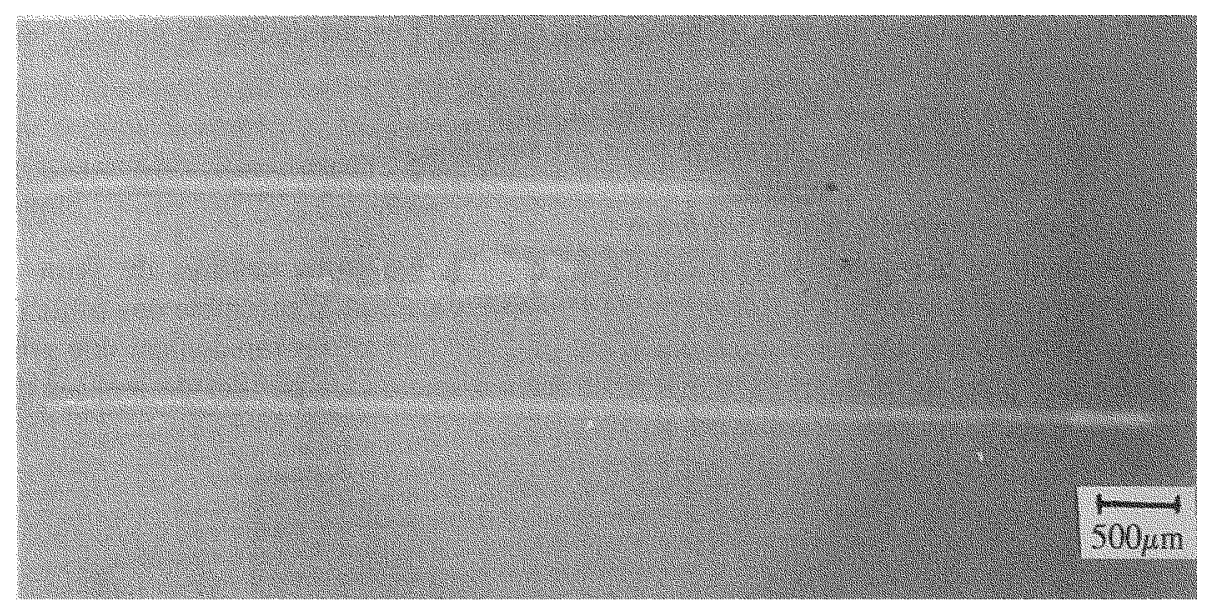

Figure 3.8: Trous débouchant et non débouchant de $100 \mu \mathrm{m}$ de diamètre dans du Quartz de $6 \mathrm{~mm}$ d'épaisseur.

De telles caractéristiques de perçage ne peuvent être obtenues par l'emploi de lasers YAG ou $\mathrm{CO}_{2}$. Les applications concernent la réalisation de buses d'injection ou de filières d'extrusion.

\section{CONCLUSION}

Le laser à excimères ouvre de nouvelles perspectives pour le micro-usinage notamment la gravure calibrée et le perçage profond sur matériaux "durs" et "ultra-durs".

Par cette technique il est possible de réaliser des usinages avec une résolution de l'ordre du micron et des rapports épaisseur sur diamètre supérieur à 10 .

Les applications sont très nombreuses et concernent toutes les industries (microélectronique, mécanique, horlogerie, optique, aéronautique, textile..).

Les sources lasers actuellement disponibles sont suffisamment fiables pour le développement d'applications industrielles et la production de pièces en séries.

Enfin, le laser à excimères est complémentaire des autres techniques de mise en forme et de transformation des matériaux (usinage mécanique, électrolytique, jet d'eau, découpe par fil, ultrasons, laser $\mathrm{YAG}$ ou $\mathrm{CO}_{2}$ ).

\section{BIBLIOGRAPHIE}

/1/F. G. Houtermans, Helv. Phys. Acta 33 (1960) 933.

12/ H. A. Koehler, L. S. Ferderber, D. L. Rehead et P. J. Ebert, Appl. Phys. Lett. 21 (1972) 198 .

13/ M. Sthele, Laser Focus World 29, 6, (1993), 135.

14/ Highlights $N^{\circ} 44$, Mai 1994.

15/ J. F. Ready "Industrial applications of lasers", Academic Press (1978). 